results, such as age, parity, and social status, failed to show any apparent bias. Furthermore, you appear to have overlooked the fact that the two studies differed in two essentials: (1) Coxon et al. investigated the use of abdominal decompression in a population of normal pregnant women and found that this had no prophylactic effect on the incidence of pre-eclampsia or the mean birth weight. We studied only those patients in whom the fetus seemed "smallfor-dates," since this is an important and not uncommon problem in obstetrics, and found that abdominal decompression seemed to have a therapeutic effect on fetal growth retardation. (2) The patients studied by Coxon et al. received abdominal decompression twice a week, whereas our patients received this treatment every day-that is, the frequency of treatment was more than three times as great in our study. The results of these two studies showed that twice-weekly decompression applied to normal pregnant patients had no prophylactic effects, while daily decompression applied to patients with fetal growth retardation had some therapeutic effects. I find nothing contradictory about these conclusions.

The final paragraph of your leading article seems to rely on reductio ad absurdum in order to arrive at the conclusion that "for most obstetricians and their patients nature's abhorrence of a vacuum seems likely to prevail." While this is elegant and epigrammatic from a literary point of view, it does little to enhance the reputation of your leading articles for expressing balanced judgements.-I am, etc.,

Westminster Medical School,

Peter Curzen

Obstetric Unit,

Queen Mary's Hospital,

1 Coxon, A., et al., fournal of Obstetrics and Gynaecology of the British Commonwealth,

2 Varma, T. R., and Curzen, P., fournal of Commonwealth, 1973, 80, 1086.

\section{Antioestrogens in Treatment of Breast} Cancer

SIR,-The paper by Drs. H. J. G. Bloom and Evelyn Boesen (6 April, p. 7) confirms the value of antioestrogens in the treatment of breast cancer. Comparison of the results of trials from different centres is notoriously difficult because of differences in the definition of response and the selection of patients. With this in mind I think it would be useful for me to elaborate on the results of the trial of tamoxifen in breast cancer carried out in Birmingham. ${ }^{1}$

It is important to note that 45 of the patients selected for treatment with nafoxidine had previously received endocrine therapy. Drs. Bloom and Boesen do not state whether a time gap was allowed between the previous endocrine treatment and the administration of nafoxidine to ensure that the response was not merely due to the rebound effect of withdrawing endocrine therapy. Similarly, in the 30 patients treated with nafoxidine who were either less than five years from the natural menopause or were treated after endocrine ablation they do not state the minimum time allowed before treatment began. If a sufficient time gap was not allowed in all patients the tumour response cannot be ascribed to the drug rather than to the endocrine ablation. No patient was admitted to the tamoxifen trial unless at least three months had elapsed since previous hormone therapy or the menopause, either natural or induced. Another difference between the trials is that the patients treated with nafoxidine presumably included some with only skeletal metastases whereas in the tamoxifen trial such patients were excluded.

As regards the definition of tumour response Drs. Bloom and Boesen comment that my criteria of objective change were less critical than theirs. I would prefer to describe my criteria as more comprehensive because I gave response data for three clearly defined groups-namely, tumour growth arrested for at least three months or tumour regression followed by regrowth within three months $9 / 68(13 \%)$, tumour size reduced to between $99 \%$ and $51 \%$ $12 / 68$ (18\%), tumour size reduced to between $50 \%$ and zero $26 / 68(38 \%)$. The third group corresponds roughly to the combined groups of "partial response" and "complete tumour response" as defined by Drs. Bloom and Boesen. For patients with advanced local tumours or nodes they observed response to nafoxidine. in $11 / 26$ $(42 \%)$. For the sake of comparison it would have been interesting if they had reported their observations for the lesser degrees of response, though one would not expect any important difference between nafoxidine and tamoxifen.

For completeness, though not mentioned in my paper, I would add that with tamoxifen the number of patients showing "arrested" disease was only $3 / 68(4 \%)$, the number showing "complete tumour regression" for at least two months was $13 / 68$ $(19 \%)$, and that if tumour response was measured at two months instead of three the number showing "partial response" was $16 / 68$ (23\%). Taking the two month criterion the combined groups at "partial response" and "complete tumour regression" showed a rate of $42 \%$ for tamoxifen-exactly the same as for nafoxidine. This suggests that as far as tumour response is concerned there is no important difference between nafoxidine and tamoxifen.

The relative importance of the side effects is more debatable. My colleagues and I have now used tamoxifen in the treatment of over 200 breast cancer patients in the Birmingham region and the previously reported response rates have been maintained. No serious side effects have been observed. For the primary endocrine management of soft tissue breast cancer (as opposed to skeletal metastases) many of us now prescribe tamoxifen in preference to the standard hormonal agents. -I am, etc.

Queen Elizabeth Hospital,

H. W. C. WARD

Birmingham

Ward, H. W. C., British Medical fournal, 1973 $1,13$.

SIR,-Drs. H. J. G. Bloom and Evelyn Boesen (6 April, p. 7) cite two different incidences for oestrogen-binding receptors$39 \%^{1}$ to agree with their $37 \%$ of postmenopausal patients deriving benefit from the antioestrogen nafoxidine, and $70 \%{ }^{2}$ to agree with the $69 \%$ of patients claimed to respond to tamoxifen. ${ }^{3}$
The mere presence of oestrogen binding does not imply that such oestrogen can then go on to promote that breast cancer or that antioestrogens will induce a regression. ${ }^{4}$ Using a test ${ }^{j}$ which reveals five different patterns of hormone dependence (and a $s, x_{\llcorner} h$, independence) and depends on finding increased enzyme activity together with improved histological appearance of the cancer tissue, we found that oestrogen promoted their breast cancer in $14 \%$ of 95 menopausas women. This agrees extremely well with the com plete tumour regression seen in 7 of the 52 patients of Drs. Bloom and Boesen.

More often in-vitro oestrogen dependence oocurs together with prolactin and growth hormone dependence, so that the use of substances which act solely as antioestrogens cannot prevent the tumours thriving on the residual pituitary hormones. This emphasizes the limitations of tests that indicate only oestrogen binding. Furthermore, the use, for example, of an androgen-iike compound as an antioestrogen could, and does in our experience, actually promote androgen-dependent tumours, so that a blind trial without knowledge of this can do harm.

Surely the time has come for trials of selected antihormonal treatment coupled with concurrent studies preselecting the six different in-vitro behaviours a ready shown by us to exist among human breast cancers.-We are, etc.,

JOHN R. HOBBS

K. A. NEWTON G. C. WESTBURY

Tumour Biology Group,

Westminster Hospital,

London S.W.I

1 Maass, H., et al., American fournal of Obstetrics and Gynecology, 1972, 113, 37

Feherty, P., Farrer-Brown, G., and Kellie, A. E., British fournal of Cancer, 1971, 25, 697,
Ward, H. W. C., British Medical fournal, 1973, 1,13 .

Braunsberg, H., et al., Lancet, 1973, 1, 163

\section{Gonorrhoea of the Pharynx}

SIR,-Your leading article (4 May, p. 239) asks for more information about the incidence of gonococcal infection of the pharynx in this country. Aware of the high incidence reported in Scandinavia, particularly in homosexuals, I have in the past two or three years made a practice of taking cultures from the pharynx of all homosexuals seen by me at James Pringle House of the Middlesex Hospital admitting to orogenital contact in the preceding month. Swabs from the pharynx have been directly inoculated on to Columbia agar plates and sugar fermentation tests carried out to distinguish the gonococcus from the other neisseriae so frequently grown.

A total of 450 pharyngeal cultures have now been obtained from these patients, but the gonococcus has been found on only eight occasions. At the time of these cultures being taken 100 of the patients had gonorrhoea elsewhere-38 urethral, 55 rectal, and seven in both sites; 59 of the patients were known to have been in contact with an identified case of gonorrhoea-40 urethral, 28 rectal, and one pharyngeal.

None of the eight patients with gonoooccal infection of the pharynx complained of a sore throat. In five of them it was the sole site of infection but the remaining three 